\title{
Predialysis hyponatremia and mortality in elderly patients beginning to undergo hemodialysis
}

\author{
Seon Ha Baek ${ }^{1,2}$, Sejoong Kim ${ }^{2,3}$, Ki Young $\mathrm{Na}^{2,3}$, Suhnggwon $\mathrm{Kim}^{4}$, and Ho Jun Chin ${ }^{2,3,4}$
}

\begin{abstract}
${ }^{1}$ Department of Internal Medicine, Hallym University Dongtan Sacred Heart Hospital, Hwaseong; ${ }^{2}$ Department of Internal Medicine, Seoul National University College of Medicine, Seoul; ${ }^{3}$ Department of Internal Medicine, Seoul National University Bundang Hospital, Seongnam; ${ }^{4}$ Research Institute of Salt and Health, Seoul, Korea
\end{abstract}

\author{
Received: September 15, 2016 \\ Revised : November 28, 2016 \\ Accepted: December 3, 2016 \\ Correspondence to \\ Ho Jun Chin, M.D. \\ Department of Internal \\ Medicine, Seoul National \\ University Bundang Hospital, \\ 82 Gumi-ro 173beon-gil, \\ Bundang-gu, Seongnam 13620, \\ Korea \\ Tel: +82-31-787-7025 \\ Fax: +82-31-787-4051 \\ E-mail: mednep@snubh.org
}

\begin{abstract}
Background/Aims: Predialysis hyponatremia has been recently reported to be associated with mortality in incident hemodialysis patients. However, whether hyponatremia is associated with unfavorable outcomes in elderly patients remains unknown. We hypothesized that nephrology referral influences hyponatremia, and aimed to define how nephrology referral affects the association between hyponatremia and mortality in the elderly.

Methods: We retrospectively assessed mortality in 599 incident hemodialysis patients aged $\geq 70$ at a tertiary university hospital, between 2000 and 2010 . We analyzed 9o-day and 1-year all-cause mortality (ACM) in relation to predialysis serum sodium (sNa). We divided the patients into two groups according to predialysis glucose-corrected sNa: hyponatremia $(<135 \mathrm{mmol} / \mathrm{L})$ and normonatremia (135 to $145 \mathrm{mmol} / \mathrm{L})$.

Results: Low estimated glomerular filtration rate, high phosphorus, low albumin, nonpreparation of arteriovenous fistula or graft, and late referral were associated with a low sNa in the elderly. Among 599 patients, 106 and 174 patients died at the 90-day and 1-year follow-ups, respectively. Each 10-mmol/L increase in predialysis sNa tended to be associated with lower 90-day and 1-year ACM. When patients were stratified by nephrology referral, hyponatremia was associated with increased mortality in early referral group (90-day ACM: hazard ratio $[\mathrm{HR}]=2.335$, $p=0.041 ; 1$-year ACM: HR $=1.790, p=0.024)$. However, hyponatremia was not associated with mortality in late referral group.

Conclusions: Predialysis hyponatremia at hemodialysis initiation is associated with late referra
\end{abstract}

Keywords: Hyponatremia; Mortality; Hemodialysis; Nephrology referral

\section{INTRODUCTION}

Hyponatremia (serum sodium $[\mathrm{sNa}]$ concentration, $<135$ $\mathrm{mmol} / \mathrm{L}$ ) is a common electrolyte disturbance among the geriatric population and is a cause of high morbidity and mortality $[1-3]$. The incidence of hyponatremia is higher in elderly patients than in younger patients because of age-associated abnormalities of water homeostasis, including changes in body composition, decrease in glomerular filtration rate (GFR), and hyper-responsiveness to arginine-vasopressin hormone with age $[3,4]$. Furthermore, elderly patients frequently use medications known to cause hyponatremia [3,5].

The incidence of hyponatremia may be high in patients with a chronic kidney disease (CKD), because these patients show a diminished ability to maintain water homeostasis $[6,7]$. Recently, a study showed that lower predialysis sNa concentration was associated with 
higher mortality in patients receiving maintenance and incident hemodialysis [8-10]. However, the relationship between predialysis hyponatremia and mortality in elderly patients beginning to undergo dialysis, who are a high-risk group for hyponatremia, has not been adequately evaluated. Therefore, we investigated the influence of hyponatremia on mortality in elderly patients undergoing incident hemodialysis.

Meanwhile, several studies have demonstrated the risk factors of hyponatremia in the dialysis population, such as malnutrition (hypoalbuminemia), lower residual renal function and estimated GFR, comorbidities, and infection [8-13]. Early nephrology referral and predialysis care was associated with the adequate control of risk factors related to CKD progression and complications, and risk factors of cardiovascular disease (i.e., nutrition, volume status, blood pressure, and anemia) [14-17]. Therefore, we hypothesize that nephrology referral affects sNa levels because predialysis care can control some of the risk factors related to hyponatremia, as discussed above. We investigated whether the timing of nephrology referral was associated with sNa concentration, and aimed to define how nephrology referral-associated hyponatremia affects the association between hyponatremia and mortality.

\section{METHODS}

\section{Study population}

The characteristics of the study population and design were published previously [18]. Between 2000 and 2010, there were 621 patients (age, $\geq 70$ years) with end-stage renal disease (ESRD) who received incident hemodialysis at Seoul National University Hospital in Korea and whose predialysis data regarding $\mathrm{sNa}$ and glucose concentrations were available. We excluded the patients with sodium levels $>145 \mathrm{mmol} / \mathrm{L}$ as their number was too small to be included $(\mathrm{n}=22)$. Consequently, $599 \mathrm{pa}-$ tients were included for this study. This study was approved by the Institutional Review Board of the Seoul National University Hospital (IRB No. H1107-092-370), with no written consent because patients records/information was anonymized and de-identified prior to analysis. All clinical investigations were conducted according to the 2008 Declaration of Helsinki and good clinical practice guidelines.

\section{Measurements and definitions}

The patients' data were retrospectively collected via a review of their electronic medical records. Laboratory data at the last time before first hemodialysis session were recorded as baseline laboratory data. Supplementary Fig. 1 shows a frequency distribution histogram of the interval between the timing of lab data and that of dialysis initiation according to nephrology referral. The concentration of sNa was corrected for serum glucose concentration by using the following formula: measured sodium concentration $+[0.016 \times$ (serum glucose concentration - 100)] [19]. According to the corrected sodium concentrations, the patients were assigned to one of the two groups of predialysis sNa concentration: hyponatremia (<135 mmol/L) and normonatremia (135 to $145 \mathrm{mmol} / \mathrm{L}$ ). Early referral (ER) and late referral (LR) were defined depending on whether the patients first consulted a nephrologist more than or less than 3 months, respectively, before they were first diagnosed with ESRD. Serum creatinine concentrations were measured using the alkaline picrate Jaffe kinetic method with an automatic analyzer (Toshiba-20oFR, Toshiba, Tokyo, Japan). Estimated GFR was calculated using the Modification of Diet in Renal Disease study equation [20]. Presence of hypertension at baseline was confirmed if the systolic blood pressure was $\geq 140 \mathrm{mmHg}$ or the diastolic blood pressure was $\geq 90 \mathrm{mmHg}$, as determined by a physical examination, or on the basis of a self-reported history of the disease or use of antihypertensive medication. Presence of diabetes mellitus was confirmed if glycated hemoglobin concentration was $\geq 6.5$, or on the basis of a self-reported history of the disease or use of antihyperglycemic agents. Information on comorbid illnesses was obtained from the International Classification of Disease, 1oth Revision (ICD-10) database at the start of hemodialysis. In this study, we transformed these ICD10 data to Charlson comorbidity index (CCI) scores using ICD-10 coding algorithm [21]. The CCI was scored at the start of hemodialysis using the definitions proposed by Charlson et al. [22].

\section{Outcome}

We combined 90-day and 1-year mortality data after dialysis initiation from Statistics Korea with our dataset, 
using each individual's unique identifier [23].

\section{Statistical analysis}

Continuous variables were expressed as mean \pm standard deviation values, and categorical variables were expressed as proportions. The concentration of sNa was presented as median (interquartile range [IQR]). Differences in continuous variables were analyzed using the Student $t$ test and Mann-Whitney $U$ test, and differences in categorical variables were analyzed using the chisquare test and Fisher exact test. The factors associated with sNa concentration were evaluated using a multivariable linear regression analysis. Cox's hazard proportion analysis was used to estimate the hazard ratios (HRs) for

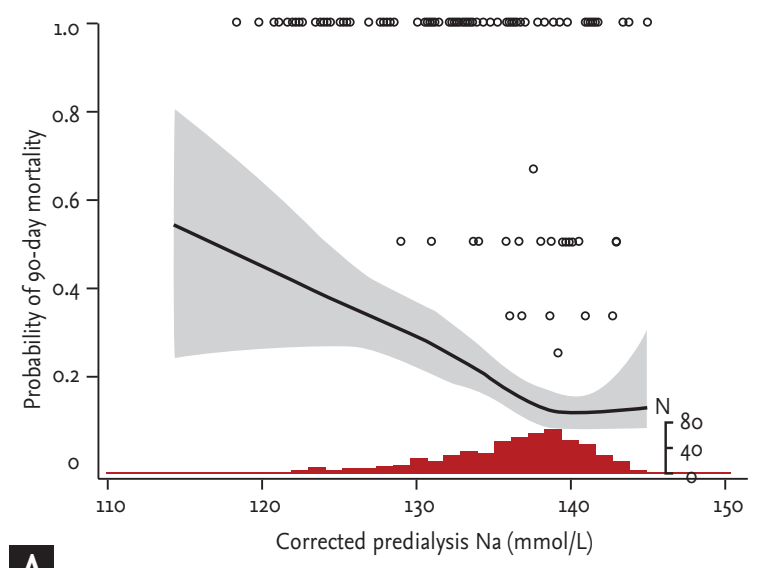

A

- Observed rate - Expected rate $-95 \% \mathrm{Cl}$ 90-day and 1-year mortality caused by hyponatremia as a continuous and categorical variables stratified by nephrology referral. We conducted the test of proportional hazards assumptions and restricted cubic spline curves (Fig. 1). The Kaplan-Meier method was used to calculate the participant survival distribution (Figs. 2 and 3). Covariate selection for the regression model was based on the significance level in univariable analysis and clinical reasoning. To prevent co-linearity among the variables, we used the backward stepwise selection method. A $p<$ 0.05 was considered statistically significant. All analyses and calculations were conducted using SPSS version 21.0 (IBM Corp., Armonk, NY, USA) and STATA version 14.0 (StataCorp LP, College Station, TX, USA).

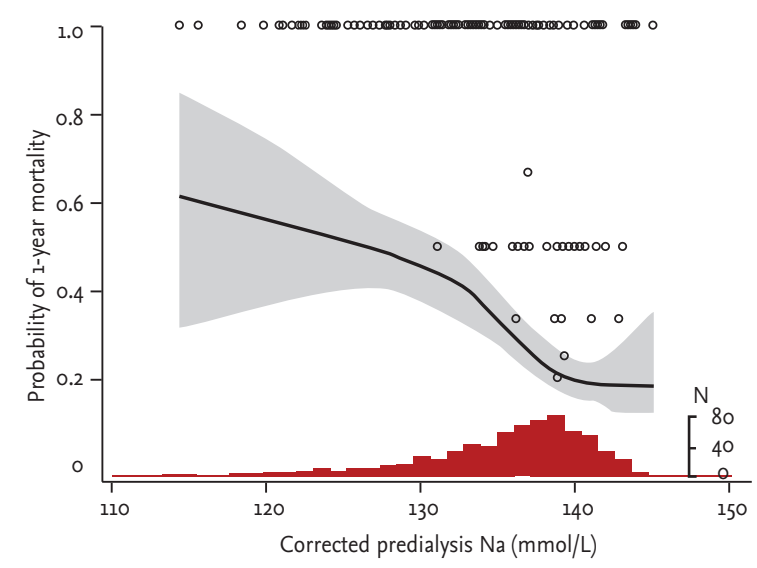

B

Figure 1. The dose-response relationship between glucose-corrected predialysis sodium and 9o-day/1-year all-cause mortality. (A) 90 -day all-cause mortality, (B) 1-year all-cause mortality. The range area indicates $95 \%$ confidence intervals (CIs). A histogram of corrected predialysis sodium is also shown.

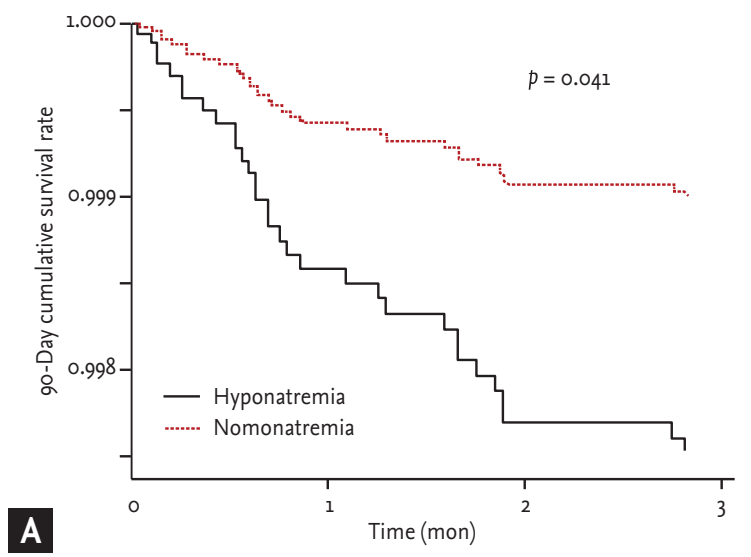

B

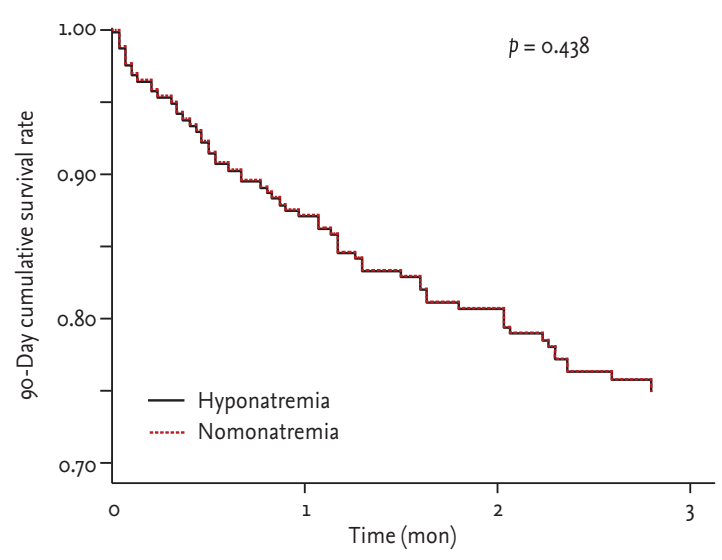

Figure 2. Kaplan-Meier survival curve for 9o-day mortality according to serum sodium (hyponatremia vs. normonatremia) stratified by nephrology referral. (A) Early referral. (B) Late referral. 

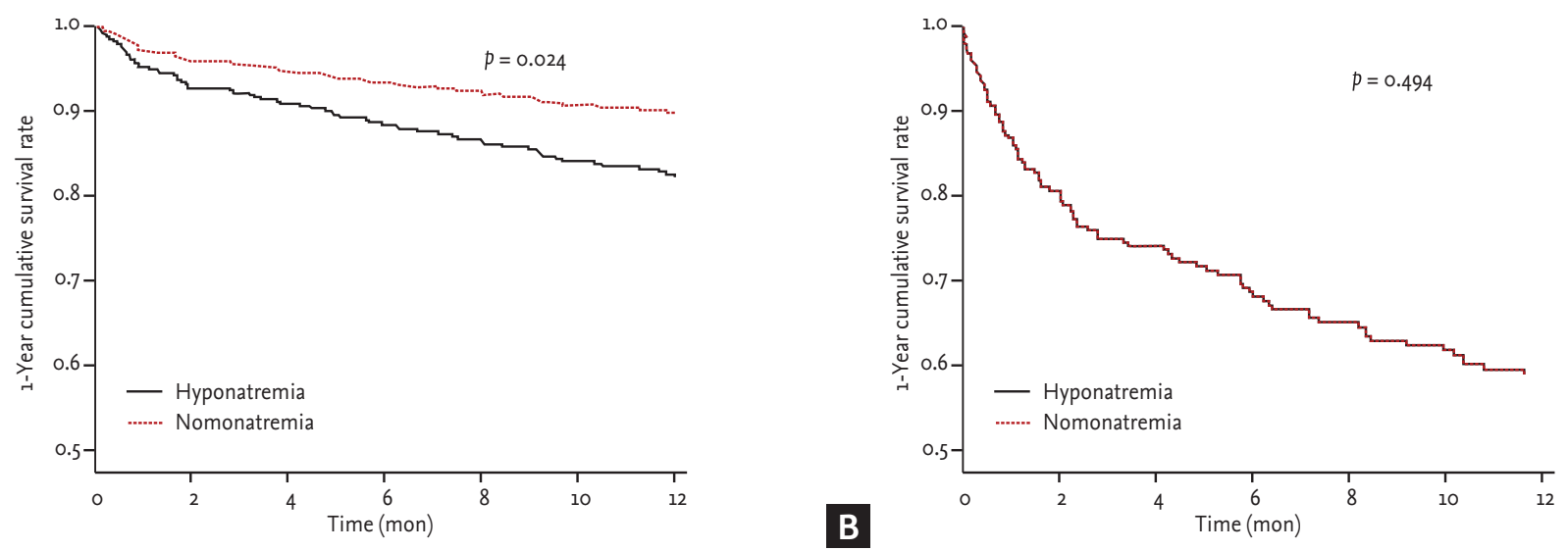

Figure 3. Kaplan-Meier survival for 1-year mortality according to serum sodium (hyponatremia vs. normonatremia) stratified by nephrology referral. (A) Early referral. (B) Late referral.

\section{RESULTS}

\section{Patients' characteristics according to the presence of hyponatremia}

The mean age of the study cohort $(\mathrm{n}=599)$ was 76.3 years, and $59.9 \%$ were men. At baseline, the median sodium concentration at dialysis initiation was $137.3 \mathrm{mmol} / \mathrm{L}$ (IQR, 133.6 to $140.0 \mathrm{mmol} / \mathrm{L}$ ) (hyponatremia [sNa < $135 \mathrm{mmol} / \mathrm{L}, \mathrm{n}=191]$; normonatremia [sNa 135 to 145 $\mathrm{mmol} / \mathrm{L}, \mathrm{n}=408])$. Among the subjects, $67.9 \%$ showed diabetes mellitus, $12.2 \%$ showed congestive heart failure (CHF), 4.0\% showed liver cirrhosis, and $18.5 \%$ showed malignancy. The mean CCI score was 5.9. In the case of most of the patients (74.4\%), hemodialysis was initiated using a central venous catheter (CVC). A comparison of the clinical characteristics according to sNa level at dialysis initiation, after stratification by the timing of nephrology referral, is presented in Table 1 and Supplementary Table 1 . Patients in the hyponatremia group ( $\mathrm{n}$ = 191) were older than patients in the normonatremia group $(\mathrm{n}=408)$. Furthermore, the frequency of use of CVC, frequency of LR at the initiation of dialysis, white blood cell (WBC) count, and serum phosphorus concentration were higher, but estimated GFR and serum albumin concentration were lower, in the hyponatremia group than in the normonatremia group (Table 1). After stratification by the timing of nephrology referral, the proportion of patients with hyponatremia was higher in the LR group than in the ER group (47.3\% vs. $22.2 \%$ ). Patients in the hyponatremia group with ER were older, had a higher WBC count, and lower estimated GFR and serum albumin level than those in the normonatremia group (Supplementary Table 1). Supplementary Fig. 2 shows a frequency distribution histogram of glucose corrected sodium concentration after stratification by the timing of nephrology referral.

\section{Factors associated with $\mathrm{s} \mathrm{Na}$ concentration}

In all of the patients, a higher sNa was associated with higher estimated GFR $(r=0.098, p=0.022)$, lower phosphorus concentration $(r=-0.116, p=0.006)$, and higher albumin concentration $(r=0.113, p=0.006)$. Furthermore, nonpreparation of arteriovenous fistula or arteriovenous graft $(\mathrm{AVF} / \mathrm{AVG})(r=-0.188, p<0.001)$, and LR $(r=-0.169, p<0.001)$ were associated with a low concentration of sodium (Table 2). History of malignancy was also associated with a low sNa concentration in the ER group (Supplementary Table 2).

\section{Ninety-day and 1-year all-cause mortality}

At the 9o-day follow-up, 55 (28.8\%) patients in the hyponatremia group, 51 patients $(12.5 \%)$ in the normonatremia group had died. Considered as a continuous predictor, each 10-mmol/L increase in the sodium concentration tended to be associated with a lower all-cause mortality $(\mathrm{HR}=0.746, p=0.122)$. The risk of 90 -day mortality was higher in the hyponatremia group than in the normonatremia group ( $\mathrm{HR}=1.547, p=0.044)$ in total. After stratification by the timing of nephrology referral, a higher sNa concentration as a continuous variable reduced the mortality $(\mathrm{HR}=0.423, p=0.016)$, and hyponatremia as a categorical variable was associated with in- 
creased mortality in the ER group $(\mathrm{HR}=2.335, p=0.041)$. However, sNa concentration or hyponatremia was not associated with mortality in the LR group (Table 3 and Fig. 2). Besides hyponatremia, nonuse of renin-angiotensin aldosterone system blockade, high phosphorus, and low albumin were associated with a significantly increased risk of 90-day mortality in the ER group $(p<$ 0.05). In the LR group, old age, higher score of CCI, and hypoalbuminemia were associated with increased risk 90-day mortality $(p<0.05)$ (Supplementary Table 3).

The 1-year overall mortality rates for patients with
ESRD were $44.0 \%$ (84 out of 191 patients) in the hyponatremia group, $22.1 \%$ (90 out of 408 patients) in the normonatremia group. A similar trend was observed when we analyzed the relationship between hyponatremia and 1-year mortality rate. The hyponatremia group also tended to showed increased 1-year mortality rate as compared to that of the normonatremia group in fully adjusted analysis ( $\mathrm{HR}=1.354, p=0.068)$. After stratification by the timing of nephrology referral, higher sNa concentration as a continuous variable reduced mortality $(\mathrm{HR}=0.593, p=0.023)$, and hyponatremia as a categor-

Table 1. Baseline characteristics of patients according to serum sodium at the time of dialysis initiation

\begin{tabular}{|c|c|c|c|c|}
\hline Characteristic & Total $(\mathrm{n}=599)$ & $\mathrm{sNa}<135(\mathrm{n}=191)$ & $\mathrm{sNa} 135-145(\mathrm{n}=408)$ & $p$ value \\
\hline Sodium level ${ }^{\mathrm{a}}, \mathrm{mmol} / \mathrm{L}$ & $137.3(133.6-140.0)$ & $131.2(127.9-133.4)$ & $139.1(137.2-141.1)$ & \\
\hline Male sex & $359(59.9)$ & $113(59.2)$ & $246(60.3)$ & 0.789 \\
\hline Age, yr & $76.3 \pm 5.0$ & $76.8 \pm 5.7$ & $76.0 \pm 4.7$ & 0.088 \\
\hline Hypertension & $592(98.8)$ & $190(99 \cdot 5)$ & $402(98.5)$ & 1.000 \\
\hline Diabetes mellitus & $401(67.9)$ & $129(68.6)$ & $272(67.5)$ & 0.850 \\
\hline Congestive heart failure & $73(12.2)$ & $28(14.7)$ & $45(11.0)$ & 0.227 \\
\hline Liver cirrhosis & $24(4.0)$ & $8(4 \cdot 2)$ & $16(3 \cdot 9)$ & 0.827 \\
\hline Malignancy & $111(18.5)$ & $39(20.4)$ & $72(17.6)$ & 0.431 \\
\hline CCI & $5.9 \pm 3.2$ & $5 \cdot 9 \pm 3.2$ & $5.9 \pm 3.1$ & 0.825 \\
\hline \multicolumn{5}{|l|}{ Medication } \\
\hline Thiazide & $60(10.0)$ & $24(12.6)$ & $36(8.8)$ & 0.188 \\
\hline Furosemide & $477(79.8)$ & $157(82.6)$ & $320(78.4)$ & 0.274 \\
\hline RAAS blockade & $198(33.1)$ & $70(36.8)$ & $128(31.4)$ & 0.192 \\
\hline$\beta$-Blocker & $428(71.6)$ & $138(72.6)$ & $290(71.1)$ & 0.770 \\
\hline Calcium channel blocker & $436(72.9)$ & $140(73.7)$ & $296(72.5)$ & 0.843 \\
\hline $\mathrm{AVF}+\mathrm{AVG} / \mathrm{CVC}$ & $150 / 435$ & $20 / 164$ & $130 / 271$ & $<0.001$ \\
\hline ER/LR & $369 / 230$ & $82 / 109$ & $287 / 121$ & $<0.001$ \\
\hline Systolic pressure, mmHg & $131.4 \pm 25.2$ & $131.2 \pm 27.1$ & $131.5 \pm 24.3$ & 0.907 \\
\hline Diastolic pressure, $\mathrm{mmHg}$ & $74.0 \pm 14.4$ & $73.2 \pm 14.8$ & $74.4 \pm 14.2$ & 0.352 \\
\hline Creatinine, $\mathrm{mg} / \mathrm{dL}$ & $6.30 \pm 2.50$ & $6.55 \pm 2.66$ & $6.18 \pm 2.42$ & 0.086 \\
\hline $\mathrm{eGFR}{ }^{\mathrm{b}}, \mathrm{mL} / \mathrm{min} / 1.73 \mathrm{~m}^{2}$ & $9.93 \pm 4.54$ & $9.45 \pm 4.36$ & $10.15 \pm 4.61$ & 0.077 \\
\hline $\mathrm{WBC}, / \mathrm{mm}^{3}$ & $8.9 \pm 5.2$ & $9.6 \pm 4.8$ & $8.5 \pm 5.4$ & 0.021 \\
\hline Hemoglobin, g/dL & $9.4 \pm 1.6$ & $9.4 \pm 1.6$ & $9.4 \pm 1.6$ & 0.789 \\
\hline Albumin, g/dL & $3.27 \pm 0.62$ & $3.11 \pm 0.62$ & $3 \cdot 35 \pm 0.61$ & $<0.001$ \\
\hline Corrected Ca, mg/dL & $8.28 \pm 0.84$ & $8.85 \pm 0.70$ & $8.86 \pm 0.77$ & 0.948 \\
\hline $\mathrm{P}, \mathrm{mg} / \mathrm{dL}$ & $5.00 \pm 1.65$ & $5.43 \pm 1.94$ & $4.81 \pm 1.45$ & $<0.001$ \\
\hline
\end{tabular}

Values are presented as median (interquartile range), number (\%), or mean $\pm \mathrm{SD}$.

sNa, serum sodium; CCI, Charlson comorbidity index; RAAS, renin angiotensin aldosterone system; AVF, arteriovenous fistula; AVG, arteriovenous graft; CVC, central venous catheter; ER, early referral; LR, late referral; eGFR, estimated glomerular filtration rate; WBC, white blood cell; Ca, calcium; P, phosphorus.

${ }^{a}$ Serum sodium was corrected for serum glucose level.

beGFR was calculated using the Modification of Diet in Renal Disease study equation. 
Table 2. Factors associated with serum sodium using multivariable linear regression

\begin{tabular}{|c|c|c|c|c|}
\hline Total & $\beta$ & Standardized $\beta$ & $95 \% \mathrm{CI}$ & $p$ value \\
\hline Age & -0.072 & -0.071 & -0.152 to 0.008 & 0.076 \\
\hline Male vs. female & 0.212 & 0.020 & -0.647 to 1.070 & 0.629 \\
\hline Hypertension & -0.712 & -0.013 & -5.008 to 3.584 & 0.745 \\
\hline Diabetes mellitus & -0.481 & -0.043 & -1.512 to 0.550 & 0.360 \\
\hline Congestive heart failure & -1.121 & -0.071 & -2.321 to 0.079 & 0.067 \\
\hline Malignancy & -0.233 & -0.018 & -1.613 to 1.147 & 0.740 \\
\hline Liver cirrhosis & 0.980 & 0.037 & -1.209 to 3.170 & 0.380 \\
\hline CCI & -0.087 & -0.037 & -0.366 to 0.191 & 0.538 \\
\hline $\mathrm{eGFR}^{\mathrm{a}}, \mathrm{mL} / \mathrm{min} / 1.73 \mathrm{~m}^{2}$ & 0.111 & 0.098 & 0.016 to 0.206 & 0.022 \\
\hline $\mathrm{CVC} / \mathrm{AVF}+\mathrm{AVG}$ & -2.201 & -0.188 & -3.157 to -1.245 & $<0.001$ \\
\hline Nephrology referral (LR/ER) & -1.812 & -0.169 & -2.686 to -0.938 & $<0.001$ \\
\hline $\mathrm{WBC}, / \mathrm{mm}^{3}$ & -0.001 & -0.001 & -0.083 to 0.081 & 0.979 \\
\hline Phosphorus, mg/dL & -0.374 & -0.116 & -0.640 to -0.107 & 0.006 \\
\hline Albumin, g/dL & 0.959 & 0.113 & 0.274 to 1.644 & 0.006 \\
\hline
\end{tabular}

CI, confidence interval; CCI, Charlson comorbidity index; eGFR, estimated glomerular filtration rate; CVC, central venous catheter; AVF, arteriovenous fistula; AVG, arteriovenous graft; LR, late referral; ER, early referral; WBC, white blood cell.

${ }^{a}$ eGFR was calculated using the Modification of Diet in Renal Disease study equation.

ical variable was associated with increased mortality in the ER group ( $\mathrm{HR}=1.790, p=0.024$ ). However, neither sodium concentration nor hyponatremia was associated with mortality in the LR group (Table 3 and Fig. 3). Besides hyponatremia, hypoalbuminemia, frequent use of CVC rather than AVF/AVG, and high phosphorus and were associated with significantly increased risk of 1 -year mortality rate in the ER group $(p<0.05)$. In the LR group, old age, hypoalbuminemia, and frequent use of CVC rather than AVF/AVG were associated with increased risk of 1 -year mortality $(p<0.05)$ (Supplementary Table 3).

\section{DISCUSSION}

In this study, among the 599 elderly patients for whom hemodialysis was initiated between 2000 and 2010, $31.8 \%$ presented with predialysis hyponatremia. We identified the factors associated with sNa concentration at dialysis initiation, and found that the timing of nephrology referral, in particular, was associated with $\mathrm{sNa}$ concentration. In addition, we found that predialysis hyponatremia increased the risk of short-term all-cause mortality in only the ER group.

In our study population, the proportion of elderly patients who experienced hyponatremia at dialysis initiation was $30.7 \%$, similar to the proportion of patients receiving acute hospital care (28.2\%) [2], and higher than the proportion of hospitalized elderly patients $(18 \%$ to $24 \%$ ) [24] or the proportion of adult outpatients receiving incident maintenance hemodialysis (12.8\%) [8]. The possible explanation for the higher prevalence of hyponatremia in our study population is that our inclusion criterion was elderly ( $\geq 70$ years) incident hemodialysis inpatients, who were at a high risk for hyponatremia. Because the definitions of the terms "elderly" and "hyponatremia" and the clinical setting vary widely among studies [3], we thought that direct comparisons of the prevalence of hyponatremia are difficult.

Our results pertaining to the factors associated with lower sNa concentrations, including lower albumin concentration, lower estimated GFR, and nonpreparation of AVF/AVG at hemodialysis initiation, were consistent with the results of the study by Nigwekar et al. [8]. In ArMORR (Incident hemodialysis patients from the Accelerated Mortality on Renal Replacement) study, hyponatremia was associated with hypercalcemia, elevated alkaline phosphatase, and hypoparathyroidism but not associated with phosphorous level [8]. However, in our study, hyperphosphatemia rather than hypercalcemia was significantly associated with hyponatremia among 
Table 3. Ninety-day and 1-year all-cause mortality risk associated with predialysis serum sodium stratified by nephrology referral

\begin{tabular}{|c|c|c|c|}
\hline Cox proportional hazard model & $\mathrm{HR}$ & $95 \% \mathrm{CI}$ & $p$ value \\
\hline \multicolumn{4}{|l|}{ 90-Day all-cause mortality } \\
\hline \multicolumn{4}{|l|}{ Total } \\
\hline Continuous model (per 10 mmol/L higher Na) & 0.746 & $0.515-1.082$ & 0.122 \\
\hline \multicolumn{4}{|l|}{ Categorical model } \\
\hline $\mathrm{sNa}<135 \mathrm{mmol} / \mathrm{L}$ & 1.547 & $1.011-2.368$ & 0.044 \\
\hline $\mathrm{sNa} \geq 135 \mathrm{mmol} / \mathrm{L}$ & 1.000 & Reference & \\
\hline \multicolumn{4}{|l|}{ Early referral } \\
\hline Continuous model (per $10 \mathrm{mmol} / \mathrm{L}$ higher $\mathrm{Na}$ ) & 0.423 & $0.210-0.854$ & 0.016 \\
\hline \multicolumn{4}{|l|}{ Categorical model } \\
\hline $\mathrm{sNa}<135 \mathrm{mmol} / \mathrm{L}$ & 2.335 & $1.037-5.261$ & 0.041 \\
\hline $\mathrm{sNa} \geq 135 \mathrm{mmol} / \mathrm{L}$ & 1.000 & Reference & \\
\hline \multicolumn{4}{|l|}{ Late referral } \\
\hline Continuous model (per 10 mmol/L higher Na) & 0.792 & $0.499-1.257$ & 0.322 \\
\hline \multicolumn{4}{|l|}{ Categorical model } \\
\hline $\mathrm{sNa}<135 \mathrm{mmol} / \mathrm{L}$ & 1.235 & $0.725-2.104$ & 0.438 \\
\hline $\mathrm{sNa} \geq 135 \mathrm{mmol} / \mathrm{L}$ & 1.000 & Reference & \\
\hline \multicolumn{4}{|l|}{ 1-Year all-cause mortality } \\
\hline \multicolumn{4}{|l|}{ Total } \\
\hline Continuous model (per 10 mmol/L higher Na) & 0.744 & $0.564-0.982$ & 0.037 \\
\hline \multicolumn{4}{|l|}{ Categorical model } \\
\hline $\mathrm{sNa}<135 \mathrm{mmol} / \mathrm{L}$ & 1.354 & $0.977-1.876$ & 0.068 \\
\hline $\mathrm{sNa} \geq 135 \mathrm{mmol} / \mathrm{L}$ & 1.000 & Reference & \\
\hline \multicolumn{4}{|l|}{ Early referral } \\
\hline Continuous model (per 10 mmol/L higher Na) & 0.593 & $0.378-0.931$ & 0.023 \\
\hline \multicolumn{4}{|l|}{ Categorical model } \\
\hline $\mathrm{sNa}<135 \mathrm{mmol} / \mathrm{L}$ & 1.790 & $1.081-2.962$ & 0.024 \\
\hline $\mathrm{sNa} \geq 135 \mathrm{mmol} / \mathrm{L}$ & 1.000 & Reference & \\
\hline \multicolumn{4}{|l|}{ Late referral } \\
\hline Continuous model (per 10 mmol/L higher Na) & 0.828 & $0.580-1.182$ & 0.299 \\
\hline \multicolumn{4}{|l|}{ Categorical model } \\
\hline $\mathrm{sNa}<135 \mathrm{mmol} / \mathrm{L}$ & 1.160 & $0.758-1.776$ & 0.494 \\
\hline $\mathrm{sNa} \geq 135 \mathrm{mmol} / \mathrm{L}$ & 1.000 & Reference & \\
\hline
\end{tabular}

Multivariable: adjusted for age, gender, hypertension, Charlson comorbidity index, nephrology referral, albumin, estimated glomerular filtration rate, phosphorus, vascular access, renin-angiotensin aldosterone system blockade, and $\beta$-blocker.

$\mathrm{HR}$, hazard ratio; CI, confidence interval; sNa, serum sodium.

index of bone mineral metabolism. Although CHF is a risk factor for hyponatremia in general, CHF was not associated with sNa concentration in our population. We thought that there was a low prevalence of heart failure in our study to demonstrate the relevance of the association between CHF and hyponatremia. Also large scale cohort studies regarding hyponatremia in ESRD showed that CHF was not always associated with hyponatremia $[8,9]$. Furthermore, late nephrology referral showed a strong, significant association with hyponatremia even in a fully adjusted model. To our knowledge, ours is the first study to show that the timing of nephrology refer- 
ral influences predialysis hyponatremia despite adjusting extensively for the possibility of confounders.

Overall, lower sNa concentration, as a continuous variable and hyponatremia, as a categorical variable tended to be associated with a higher risk of mortality in incident elderly hemodialysis patients. Although the mechanism(s) are not entirely clear, $\mathrm{sNa}$ concentration influences the 3-dimensional conformations of protein and enzyme and plays a critical role in cellular function. The relationship between abnormal sNa and cerebral dysfunction has been well described $[10,25]$. However, it remains uncertain whether hyponatremia is a bystander or a causative factor. While one meta-analysis showed that improvement in hyponatremia would revert the mortality risk related to hyponatremia [26], some other studies demonstrated that the resolution of hyponatremia did not reduce this risk [27-29]. We thought that hypona tremia might be used to predict for mortality. When the patient data were analyzed separately after stratification by the timing of nephrology referral, hyponatremia predicted mortality in only the ER group. No impact of hyponatremia or lower predialysis sNa concentration on mortality was observed in the LR group. We also re-conducted analysis, based on the patient's with laboratory data immediately before dialysis (the ones with "o to 1 day," $n=440$ ). We rigorously controlled for the influence of the available laboratory data on patients. A similar trend was observed when we analyzed the relationship between hyponatremia and 90-day/1year mortality rate (90-day mortality: $\mathrm{HR}=2.367, p=$ 0.037 in ER group, HR = 1.280, $p=0.384$ in LR group; 1-year mortality: $\mathrm{HR}=1.8 \mathrm{O} 2, p=0.040$ in ER group, HR $=1.216, p=0.401$ in LR group). The possible explanations for the difference in the impact of hyponatremia on survival according to the timing of nephrology referral are as follows: first, in our data, although LR group experienced chronic inflammation and malnutrition more frequently (higher WBC and lower albumin concentration) compared to ER group, only ER group have statistically significant differences in WBC and albumin which were well-known to be predictors for mortality in incident hemodialysis patients between hyponatremia and normonatremia (Supplementary Table 1) [30,31]. Second, LR group experienced acute complications more frequently during the early period of dialysis, including infection and cardiovascular events, contributing to higher early-period mortality rate [18,32-36], although we could not confirm this possibility through our data because the cause of death was unavailable. In the present study, other factors including chronic or acute inflammation (i.e., frequent CVC use) or malnutrition (hypoalbuminemia) might have overshadowed hyponatremia as a predictor for 90-day and 1-year mortality in the LR group (Supplementary Table 3). We thought that referral-associated other acute complications, rather than hyponatremia, affected mortality in the LR group. We emphasize the originality of this study in that predialysis hyponatremia affected early-period mortality in the elderly incident hemodialysis patients, and in particular, nephrology referral associated hyponatremia affected the association between hyponatremia and mortality.

This study has several limitations. First, we used only a single sNa concentration for the analysis. Repeated measures of sNa concentration may reflect the true internal condition and yield more accurate results. Second, other confounding factors, including ultrafiltration volume, dialysate sodium concentration, residual renal function, and Kt/V cannot be excluded. However, data regarding dialysis prescription were unavailable. Previous studies have emphasized on vascular access, predialysis care, and nutritional status (hypoalbuminemia) rather than dialysis-associated factors as predictors of early-period mortality $[34,36]$. The dialysate sodium concentration is not typically adjusted for the predialysis sodium concentrations [10]. Third, although previous studies have clarified the effects of hyponatremia on cellular function or organ system [25,33], our retrospective study could not clearly explain the mechanism by which hyponatremia affects mortality.

In conclusion, predialysis hyponatremia at the initiation of maintenance hemodialysis is associated with the timing of nephrology referral and increased risk of mortality in the elderly. In particular, predialysis hyponatremia increased the risk of short-term all-cause mortality in only the ER group, and nephrology referral associated affected the association between hyponatremia and mortality in the elderly. Future prospective studies will be needed to determine whether an abnormal sNa concentration should be considered as an indication for treatment in clinical trials. 


\section{KEY MESSAGE}

1. Predialysis hyponatremia at hemodialysis initiation is associated with the timing of nephrology referral and increased risk of short-term mortality in the elderly, particularly among early referral group.

2. Nephrology referral affects the association between hyponatremia and mortality in elderly patients.

\section{Conflict of interest}

No potential conflict of interest relevant to this article was reported.

\section{REFERENCES}

1. Upadhyay A, Jaber BL, Madias NE. Incidence and prevalence of hyponatremia. Am J Med 2006;119:S30-S35.

2. Hawkins RC. Age and gender as risk factors for hyponatremia and hypernatremia. Clin Chim Acta 2003;337:169172.

3. Shapiro DS, Sonnenblick M, Galperin I, Melkonyan L, Munter G. Severe hyponatraemia in elderly hospitalized patients: prevalence, aetiology and outcome. Intern Med J 2010;40:574-580.

4. Cowen LE, Hodak SP, Verbalis JG. Age-associated abnormalities of water homeostasis. Endocrinol Metab Clin North Am 2013;42:349-370.

5. Wilkinson TJ, Begg EJ, Winter AC, Sainsbury R. Incidence and risk factors for hyponatraemia following treatment with fluoxetine or paroxetine in elderly people. Br J Clin Pharmacol 1999;47:211-217.

6. Kovesdy CP, Lott EH, Lu JL, et al. Hyponatremia, hypernatremia, and mortality in patients with chronic kidney disease with and without congestive heart failure. Circulation 2012;125:677-684.

7. Spasovski G, Vanholder R, Allolio B, et al. Clinical practice guideline on diagnosis and treatment of hyponatraemia. Eur J Endocrinol 2014;170:G1-G47.

8. Nigwekar SU, Wenger J, Thadhani R, Bhan I. Hyponatremia, mineral metabolism, and mortality in incident maintenance hemodialysis patients: a cohort study. Am J Kidney Dis 2013;62:755-762.

9. Hecking M, Karaboyas A, Saran R, et al. Predialysis serum sodium level, dialysate sodium, and mortality in maintenance hemodialysis patients: the Dialysis Outcomes and Practice Patterns Study (DOPPS). Am J Kidney Dis 2012;59:238-248.

10. Waikar SS, Curhan GC, Brunelli SM. Mortality associated with low serum sodium concentration in maintenance hemodialysis. Am J Med 2011;124:77-84.

11. Xu R, Pi HC, Xiong ZY, et al. Hyponatremia and cognitive impairment in patients treated with peritoneal dialysis. Clin J Am Soc Nephrol 2015;10:1806-1813.

12. Mandai S, Kuwahara M, Kasagi Y, et al. Lower serum sodium level predicts higher risk of infection-related hospitalization in maintenance hemodialysis patients: an observational cohort study. BMC Nephrol 2013;14:276.

13. Dimitriadis C, Sekercioglu N, Pipili C, Oreopoulos D, Bargman JM. Hyponatremia in peritoneal dialysis: epidemiology in a single center and correlation with clinical and biochemical parameters. Perit Dial Int 2014;34:260270.

14. Herget-Rosenthal S, Quellmann T, Linden C, Hollenbeck M, Jankowski V, Kribben A. How does late nephrological co-management impact chronic kidney disease? An observational study. Int J Clin Pract 2010;64:1784-1792.

15. Arora P, Obrador GT, Ruthazer R, et al. Prevalence, predictors, and consequences of late nephrology referral at a tertiary care center. J Am Soc Nephrol 1999;10:1281-1286.

16. Stack AG. Impact of timing of nephrology referral and pre-ESRD care on mortality risk among new ESRD patients in the United States. Am J Kidney Dis 2003;41:310318.

17. Lorenzo V, Martn M, Rufino M, Hernandez D, Torres A, Ayus JC. Predialysis nephrologic care and a functioning arteriovenous fistula at entry are associated with better survival in incident hemodialysis patients: an observational cohort study. Am J Kidney Dis 2004;43:999-1007.

18. Baek SH, Ahn Sy, Lee SW, et al. Outcomes of predialysis nephrology care in elderly patients beginning to undergo dialysis. PLoS One 2015;10:e0128715.

19. Katz MA. Hyperglycemia-induced hyponatremia: calculation of expected serum sodium depression. N Engl J Med 1973;289:843-844.

20. Levey AS, Coresh J, Greene T, et al. Using standardized serum creatinine values in the modification of diet in renal disease study equation for estimating glomerular filtration rate. Ann Intern Med 2006;145:247-254.

21. Chae JW, Song CS, Kim H, Lee KB, Seo BS, Kim DI. Pre- 
diction of mortality in patients undergoing maintenance hemodialysis by Charlson comorbidity index using ICD10 database. Nephron Clin Pract 2011;117:c379-c384.

22. Charlson ME, Pompei P, Ales KL, MacKenzie CR. A new method of classifying prognostic comorbidity in longitudinal studies: development and validation. J Chronic Dis 1987;40:373-383.

23. Statistics Korea. MDIS: Microdata Integrated Service [Internet]. Daejeon (KR): Statistics Korea [cited 2017 Sep 18]. Available from: https://mdis.kostat.go.kr/index.do.

24. Hoyle GE, Chua M, Soiza RL. Prevalence of hyponatremia in elderly patients. J Am Geriatr Soc 2006;54:1473.

25. Renneboog B, Musch W, Vandemergel X, Manto MU, Decaux G. Mild chronic hyponatremia is associated with falls, unsteadiness, and attention deficits. Am J Med 2006;119:71.

26. Corona G, Giuliani C, Verbalis JG, Forti G, Maggi M, Peri A. Hyponatremia improvement is associated with a reduced risk of mortality: evidence from a meta-analysis. PLoS One 2015;10:e0124105.

27. Lee SE, Choi DJ, Yoon CH, et al. Improvement of hyponatraemia during hospitalisation for acute heart failure is not associated with improvement of prognosis: an analysis from the Korean Heart Failure (KorHF) registry. Heart 2012;98:1798-1804.

28. Gheorghiade M, Rossi JS, Cotts W, et al. Characterization and prognostic value of persistent hyponatremia in patients with severe heart failure in the ESCAPE Trial. Arch Intern Med 2007;167:1998-2005.

29. Waikar SS, Mount DB, Curhan GC. Mortality after hospi- talization with mild, moderate, and severe hyponatremia. Am J Med 2009;122:857-865.

30. Hsu CW, Lin JL, Lin-Tan DT, Yen TH, Chen KH. White blood cell count predicts all-cause, cardiovascular disease-cause and infection-cause one-year mortality of maintenance hemodialysis patients. Ther Apher Dial 2010;14:552-559.

31. Wagner M, Ansell D, Kent DM, et al. Predicting mortality in incident dialysis patients: an analysis of the United Kingdom Renal Registry. Am J Kidney Dis 2011;57:894902.

32. Collins AJ, Foley RN, Gilbertson DT, Chen SC. The state of chronic kidney disease, ESRD, and morbidity and mortality in the first year of dialysis. Clin J Am Soc Nephrol 2009;4 Suppl 1:S5-S11.

33. Lukowsky LR, Kheifets L, Arah OA, Nissenson AR, Kalantar-Zadeh K. Patterns and predictors of early mortality in incident hemodialysis patients: new insights. Am J Nephrol 2012;35:548-558.

34. McQuillan R, Trpeski L, Fenton S, Lok CE. Modifiable risk factors for early mortality on hemodialysis. Int $\mathrm{J}$ Nephrol 2012;2012:435736.

35. Chan KE, Maddux FW, Tolkoff-Rubin N, Karumanchi SA, Thadhani R, Hakim RM. Early outcomes among those initiating chronic dialysis in the United States. Clin J Am Soc Nephrol 2011;6:2642-2649.

36. Bradbury BD, Fissell RB, Albert JM, et al. Predictors of early mortality among incident US hemodialysis patients in the Dialysis Outcomes and Practice Patterns Study (DOPPS). Clin J Am Soc Nephrol 2007;2:89-99. 


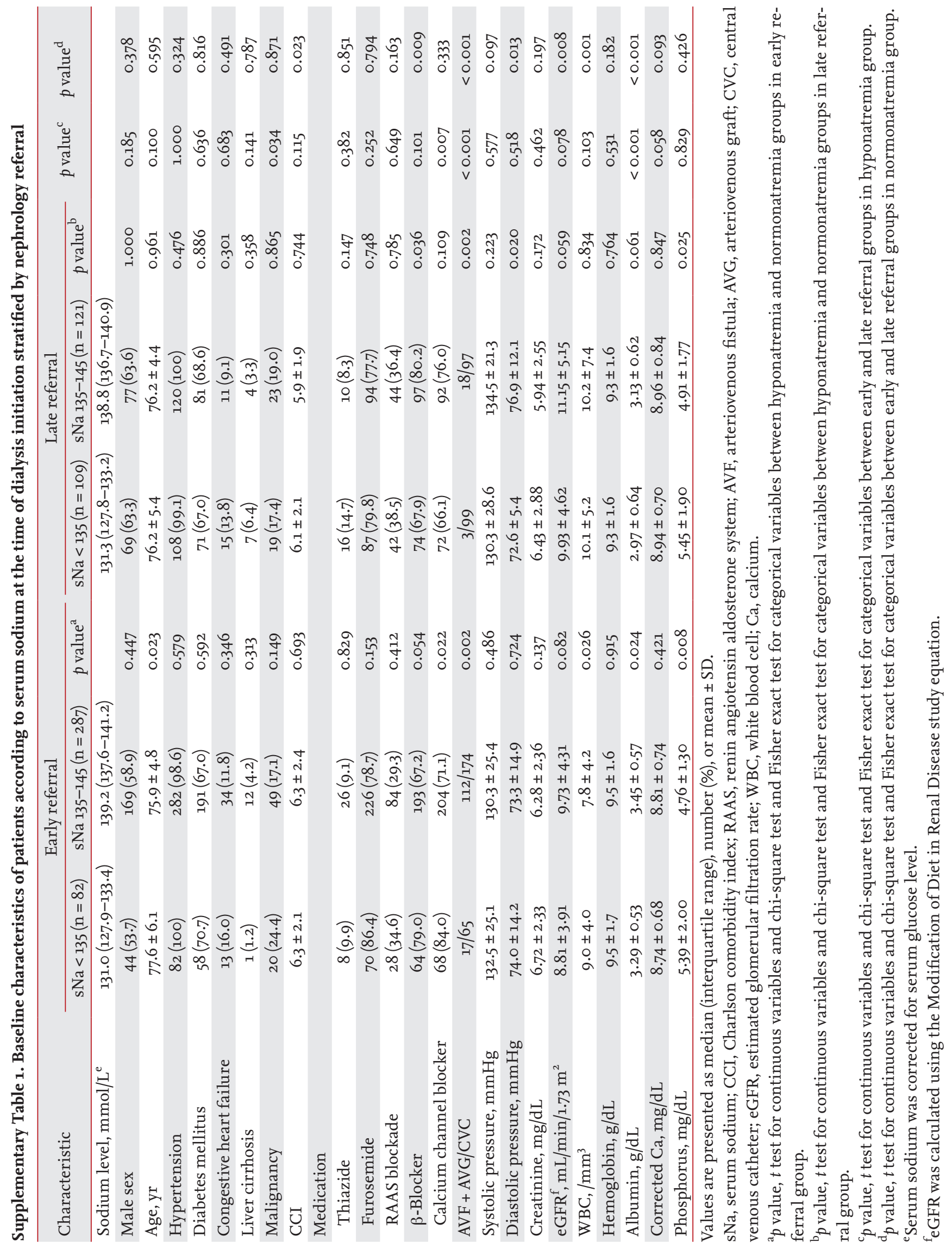


Baek $\mathrm{SH}$, et al. $\mathrm{Na}$ and mortality in elderly hemodialysis

Supplementary Table 2. Factors associated with serum sodium using multivariable linear regression stratified by nephrology referral

\begin{tabular}{|c|c|c|c|c|}
\hline Variable & $\beta$ & Standardized $\beta$ & $95 \% \mathrm{CI}$ & $p$ value \\
\hline \multicolumn{5}{|l|}{ Early referral } \\
\hline Age & -0.110 & -0.118 & -0.205 to -0.015 & 0.023 \\
\hline Malignancy & -1.266 & -0.104 & -2.463 to -0.069 & 0.038 \\
\hline $\mathrm{eGFR}^{\mathrm{a}}, \mathrm{mL} / \mathrm{min} / 1.73 \mathrm{~m}^{2}$ & $0.15^{8}$ & 0.139 & 0.036 to 0.279 & 0.011 \\
\hline $\mathrm{CVC} / \mathrm{AVF}+\mathrm{AVG}$ & -2.234 & -0.223 & -3.244 to -1.223 & $<0.001$ \\
\hline Albumin, g/dL & 0.903 & 0.107 & 0.053 to 1.753 & 0.037 \\
\hline Phophorus, mg/dL & -0.349 & -0.109 & -0.688 to -0.009 & 0.044 \\
\hline \multicolumn{5}{|l|}{ Late referral } \\
\hline CVC/AVF + AVG & -2.633 & -0.148 & -5.001 to -0.265 & 0.029 \\
\hline CCI & -0.415 & -0.155 & -0.816 to -0.015 & 0.042 \\
\hline Phophorus, mg/dL & -0.459 & -0.151 & -0.863 to -0.055 & 0.026 \\
\hline
\end{tabular}

Multivariable: age, gender, hypertension, diabetes mellitus, congestive heart failure, malignancy, liver cirrhosis, charlson comorbidity index, eGFR, vascular access, nephrology referral, white blood cell, phosphorus, and albumin.

CI, confidence interval; eGFR, estimated glomerular filtration rate; CVC, central venous catheter; AVF, arteriovenous fistula; AVG, arteriovenous graft; CCI, Charlson comorbidity index.

${ }^{a}$ eGFR was calculated using the Modification of Diet in Renal Disease study equation. 
Supplementary Table 3. Factors associated with 90 -day and 1-year mortality using multivariable cox regression stratified by nephrology referral

\begin{tabular}{|c|c|c|c|}
\hline Variable & HR & $95 \% \mathrm{CI}$ & $p$ value \\
\hline \multicolumn{4}{|l|}{ 9०-Day mortality } \\
\hline \multicolumn{4}{|l|}{ Early referral } \\
\hline Hyponatremia vs. Normonatremia & 2.335 & $1.037-5.261$ & 0.041 \\
\hline Use of RAAS blockade & 0.368 & $0.154-0.882$ & 0.025 \\
\hline Albumin, g/dL & 0.504 & $0.258-0.985$ & 0.045 \\
\hline Phosphorus, mg/dL & 1.490 & $1.224-1.814$ & $<0.001$ \\
\hline \multicolumn{4}{|l|}{ Late referral } \\
\hline Age & 1.083 & $1.033-1.136$ & 0.001 \\
\hline CCI & 1.132 & $1.003-1.277$ & 0.045 \\
\hline Albumin, g/dL & 0.507 & $0.332-0.774$ & 0.002 \\
\hline \multicolumn{4}{|l|}{ 1-Year mortality } \\
\hline \multicolumn{4}{|l|}{ Early referral } \\
\hline Hyponatremia vs. Normonatremia & 1.790 & $1.081-2.962$ & 0.024 \\
\hline Albumin, g/dL & 0.667 & $0.453-0.982$ & 0.040 \\
\hline $\mathrm{CVC} / \mathrm{AVF}+\mathrm{AVG}$ & 10.560 & $3.289-33.901$ & $<0.001$ \\
\hline Phosphorus, mg/dL & 1.202 & $1.049-1.376$ & 0.008 \\
\hline \multicolumn{4}{|l|}{ Late referral } \\
\hline Age & 1.066 & $1.024-1.110$ & 0.002 \\
\hline Albumin, g/dL & 0.450 & $0.315-0.642$ & $<0.001$ \\
\hline $\mathrm{CVC} / \mathrm{AVF}+\mathrm{AVG}$ & 6.345 & $1.554-25.903$ & 0.010 \\
\hline
\end{tabular}

Multivariable, adjusted for age, gender, hypertension, Charlson comorbidity index, nephrology referral, albumin, estimated glomerular filtration rate ${ }^{a}$, phosphorus, vascular access, renin-angiotensin aldosterone system blockade, and $\beta$-blocker. $\mathrm{HR}$, hazard ratio; CI, confidence interval; RAAS blockade, renin-angiotensin aldosterone system blockade; CCI, Charlson comorbidity index; CVC, central venous catheter; AVF, arteriovenous fistula; AVG, arteriovenous graft.

${ }^{a}$ Estimated glomerular filtration rate was calculated using the Modification of Diet in Renal Disease study equation. 

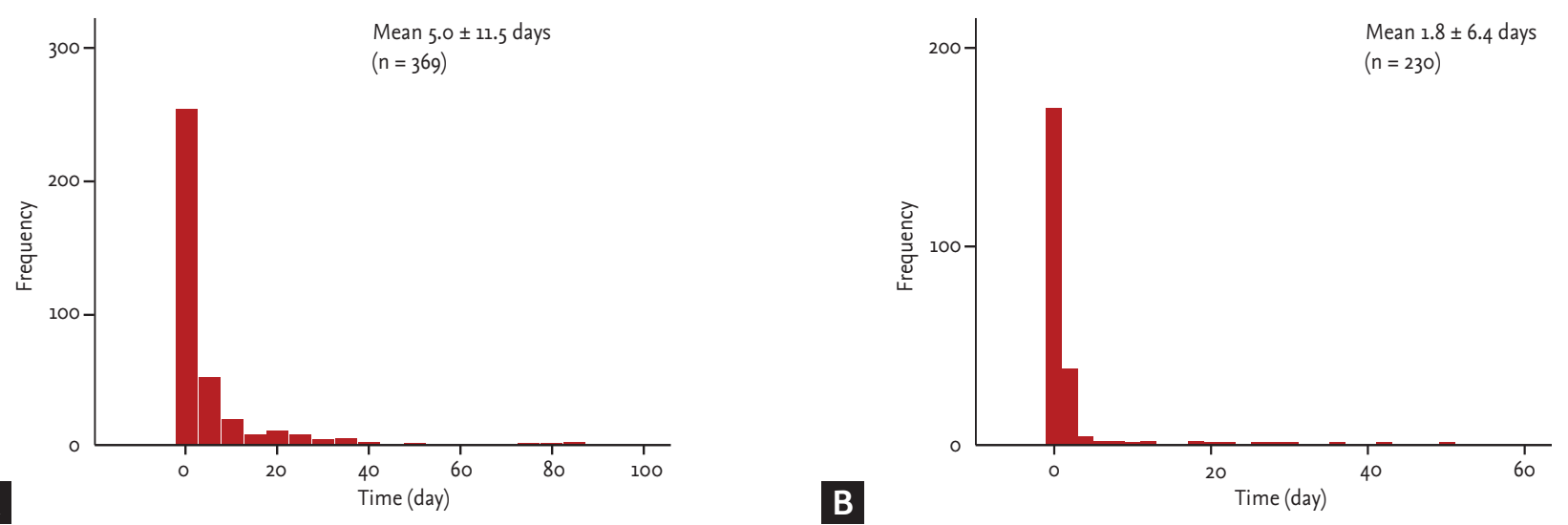

Supplementary Figure 1. A frequency distribution histogram of the interval between the timing of lab data and that of dialysis initiation according to nephrology referral. (A) Early referral. (B) Late referral. 


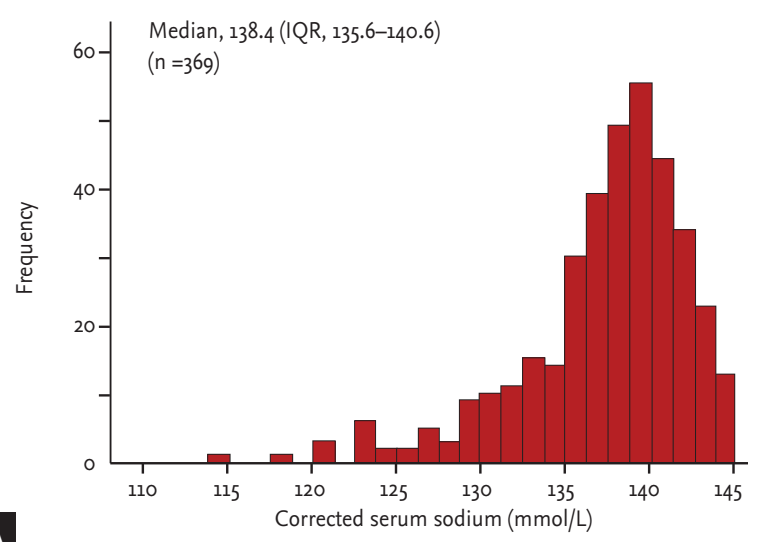

A

Supplementary Figure 2. A distribution of glucose-corrected serum sodium according to nephrology referral. (A) Early referral. (B) Late referral. IQR, interquartile range. 